\title{
Disorder effect on the magnetic behavior of mechanically alloyed $\mathrm{Fe}_{1-x} \mathrm{Al}_{x}(0.2 \leqslant x \leqslant 0.4)$
}

\author{
Ligia E. Zamora, ${ }^{1,2}$ G. A. Pérez Alcázar ${ }^{1,2}$ G. Y. Vélez, ${ }^{1}$ J. D. Betancur, ${ }^{1}$ J. F. Marco, ${ }^{3}$ J. J. Romero,,${ }^{4,5}$ A. Martínez, ${ }^{4}$ \\ F. J. Palomares, ${ }^{6}$ and J. M. González ${ }^{2}$ \\ ${ }^{1}$ Departamento de Física, Universidad del Valle, A.A. 25360 Cali, Colombia \\ ${ }^{2}$ Unidad Asociada ICMM-IMA, c/Sor Juana Inés de la Cruz, 3, 28049 Madrid, Spain \\ ${ }^{3}$ Instituto de Química-Física “Rocasolano," CSIC, c/Serrano 119, 28006 Madrid, Spain \\ ${ }^{4}$ Instituto de Magnetismo Aplicado, P.O. Box 155, Las Rozas, 28230 Madrid, Spain \\ ${ }^{5}$ Instituto de Cerámica y Vidrio, CSIC, C/Kelsen 5, 28049 Madrid, Spain \\ ${ }^{6}$ Instituto de Ciencia de Materiales de Madrid-CSIC, c/Sor Juana Inés de la Cruz, 3, 28049 Madrid, Spain
}

(Received 29 October 2008; revised manuscript received 18 December 2008; published 20 March 2009)

\begin{abstract}
We report on the magnetic properties of $\mathrm{Fe}_{1-x} \mathrm{Al}_{x}$ alloys $(0.2 \leqslant x \leqslant 0.4)$ produced by mechanical alloying by milling pure element powders for $t=12,24$, and $36 \mathrm{~h}$. The alloys present a bcc lattice with compositional disorder and are ferromagnetic at room temperature, independently of the milling time. The lattice parameter of the $x=0.2$ sample presents a small decrease with $t$, whereas those of the $x=0.3$ and 0.4 samples remain constant independently of the milling time. The magnetic properties of the alloys with $x=0.2$ and 0.3 do not show important variations with $t$, while those of $x=0.4$ are strongly dependent on the milling time. For this latter alloy it was found that: (i) despite being the most diluted of the series, it presents a well developed ferromagnetic order at room temperature as the Mössbauer and hysteretic data have shown; (ii) the temperature dependence of the ac susceptibility and the Mössbauer spectra recorded at different temperatures evidence the occurrence of reentrant spin-glass and superparamagnetic phenomena. The enhancement of the ferromagnetic behavior and the presence of reentrant spin-glass freezing temperature and of a superparamagnetic blocking process are interpreted in terms of a simple localized model based on the disorder present in that alloy and on the occurrence of competitive interactions, namely, the ferromagnetic nearest-neighbor Fe-Fe interactions and the antiferromagnetic near-nearest-neighbor Fe-Fe ones. Taken together, these results evidence that the stabilization of the magnetic order takes place in the $x=0.4$ sample exclusively through the induction of compositional disorder and without any contribution from the lattice expansion.
\end{abstract}

DOI: 10.1103/PhysRevB.79.094418

PACS number(s): 75.50.Bb, 75.50.Lk, 71.20.Lp, 76.80.+y

\section{INTRODUCTION}

The Fe-Al alloys form a complete solid solution series that has been the subject of a large deal of research efforts due to (i) the rich variety of magnetic order types observable in the series, ${ }^{1-11}$ (ii) the relevant role of disorder in the occurrence of those magnetic phases, ${ }^{1,2,4,8,10-13}$ (iii) the observation of expansion effects whose link to the induction of magnetic order is not completely established, ${ }^{8,16-21}$ and (iv) the applicability of these alloys as structural and corrosion/ oxidation resistant materials.

Besides the ferromagnetic $(\mathrm{F})$ and paramagnetic $(\mathrm{P})$ phases observed at room temperature (RT) in the Fe-Al series, two additional magnetic order phenomenologies have been also reported. The first one is the occurrence of spinglass (SG) phases which were initially observed in ordered alloys with compositions ranging from 25 to 30 at. $\% \mathrm{Al}$ as related to an anomalous behavior on temperature dependence of the average hyperfine field, $\left\langle B_{\mathrm{hf}}\right\rangle$, and the mean magnetic moment, $\langle\mu\rangle{ }^{1,2}$ Experimental and theoretical evidences have established that this anomaly corresponds to a pure SG freezing transition, ${ }^{4-6}$ which takes place at temperatures near $30 \mathrm{~K}$. For ordered $\mathrm{Fe}_{0.7} \mathrm{Al}_{0.3}$ single crystals, this transition was reported to be of the re-entrant spin-glass (RSG) type with a freezing temperature of $\sim 80 \mathrm{~K} .{ }^{7,8}$ The second phenomenon is the superparamagneticlike (SP) behavior initially proposed by Cable et al. ${ }^{10}$ for alloys with a composition around 30 at. \% Al. That cluster occurrence has been in- voked for some authors to interpret the evolution with the temperature of the magnetic properties of the $\mathrm{Fe}-\mathrm{Al}$ disordered alloys. ${ }^{10-12}$

The properties of the $\mathrm{F}$ phases present in the $\mathrm{Fe}-\mathrm{Al}$ alloys are strongly dependent both on their composition and on their fabrication details. In the case of the melted alloys it was reported that for $\mathrm{Al}$ contents up to 18 at. \% the samples (of the $A_{2}$ structural type) are $\mathrm{F}$ and disordered at RT. For Al contents in the range from 18 to 51 at. \%, samples melted and quenched from a postpreparation treatment at $700{ }^{\circ} \mathrm{C}$ correspond to the nonstoichiometric ordered type $B_{2}$, whereas samples with contents in the range from 33 to 51 at. \% $\mathrm{Al}$, which were slowly cooled from a treatment at $700{ }^{\circ} \mathrm{C}$, correspond to the nonstoichiometric $\mathrm{DO}_{3}$ type..$^{1-4}$

A well-known result ${ }^{1,3,23}$ about the phenomenology linked to the induction of disorder in this series is that related to the RT cold work of the samples upon which become structurally disordered, whereas their F order is stabilized if it is present (the RT saturation magnetization and/or the order temperature increase) or is induced if it is absent. This enhancement of the $\mathrm{F}$ order associated to the structural disorder was reported in melted alloys, treated at $1000^{\circ} \mathrm{C}$ and quenched in ice water which became disordered so as to allow the observation of RT F up to 47.5 at. \% $\mathrm{Al}^{24}$ This phenomenology has been also observed in alloys prepared by sputtering ${ }^{25}$ and implantation techniques. ${ }^{26}$ Recently, Fe-Al disordered alloys have been also produced by mechanical alloying (MA). ${ }^{27}$ In these samples the occurrence of RT F 
behavior dependent on the milling conditions has been reported for $\mathrm{Al}$ contents above 33 at. \%.

Besides the work of Bogner et al. ${ }^{13}$ in which the Mössabauer spectra taken in a $\mathrm{Fe}_{0.5} \mathrm{Al}_{0.5}$ single crystal were analyzed in terms of band structure calculations and of a thermodynamic model, Reddy et al., ${ }^{14}$ by using electronic structure models for FeAl clusters, showed that they are highly magnetic and that their ground state is dominated by the Fe-Fe bonds, differently from the ordered bulk $\mathrm{Fe}_{0.5} \mathrm{Al}_{0.5}$ alloy that is nonmagnetic at RT and is dominated by the Fe-Al bonds. Reddy et al. ${ }^{14}$ also proposed that $\mathrm{Fe}_{0.5} \mathrm{Al}_{0.5}$ nanoparticles are $\mathrm{F}$ due to the $\mathrm{Fe}$ sites at the surface which present large moments originated by their reduced coordination. Apiñaniz et al. ${ }^{15}$ using a self-consistent electronic calculation showed that the induction of disorder in the $\mathrm{Fe}_{0.5} \mathrm{Al}_{0.5}$ and $\mathrm{Fe}_{0.75} \mathrm{Al}_{0.25}$ alloys increases the mean magnetic moment with respect to that measurable in the ordered ones, this increase being larger in the case of the $\mathrm{Fe}_{0.5} \mathrm{Al}_{0.5}$ alloy. Das et al. ${ }^{16}$ using self-consistent electronic calculations based on the tight-binding linear muffin-tin orbital method showed, for the intermetallics $\mathrm{Fe}_{1-x} \mathrm{Al}_{x}$ with $0 \leqslant x \leqslant 0.5$, that the $\mathrm{Fe}_{0.5} \mathrm{Al}_{0.5}$ has two nearly degenerated states: a nonmagnetic state with zero moment on $\mathrm{Fe}$ and a $\mathrm{F}$ state with a moment of $0.75 \mu_{B}$ per $\mathrm{Fe}$ atom, and that the $\mathrm{Fe}_{3} \mathrm{Al}$ is $\mathrm{F}$ with a calculated moment of $2.45 \mu_{B}$ at the Fe-I site and $1.95 \mu_{B}$ at the Fe-II site. The bonding between the $\mathrm{Fe}$ and $\mathrm{Al}$ atoms is antiferromagnetic (AF) and is primarily due to hybridization between the $3 d$ electrons of the former and the $s p$ electrons of the latter.

Complementing the above summarized results about the influence of disorder on the enhancement of the magnetic order, another well-known experimental fact is the expansion effect produced, as a consequence of its larger atomic size, when $\mathrm{Al}$ substitute $\mathrm{Fe}$ atoms in the bcc lattice. This expansion was reported in ordered alloys produced by melting, ${ }^{28}$ when they were mechanically grinded (MG) and then heat treated, ${ }^{17-22}$ as well as in disordered melted alloys. ${ }^{23}$ According to recent literature, ${ }^{10,16-21}$ the disorder and the lattice expansion observed in MG samples are jointly responsible for the enhancement of the F behavior and these effects are more notable for samples in the region between 40 and 50 at. $\% \mathrm{Al}$.

The present work aims at the characterization of the structural and magnetic properties of the $\mathrm{Fe}_{1-x} \mathrm{Al}_{x}(0.2 \leqslant x \leqslant 0.4)$ alloy samples produced by MA using different milling times. That study has been carried out by means of x-ray diffraction (XRD), vibrating-sample magnetometry (VSM), ac magnetic susceptibility, and Mössbauer spectrometry (MS) and, from the obtained results, we propose a simple ordered and disordered localized atomic picture giving account of the $\mathrm{F}$ enhancement and of the occurrence of the SG and SP phenomena in this system.

\section{EXPERIMENTAL PROCEDURE}

In this work we have prepared $\mathrm{Fe}_{1-x} \mathrm{Al}_{x}$ alloys, with compositions in the range $0.2 \leqslant x \leqslant 0.4$, by mechanical alloying using high purity (more than 99.9\%) Fe and $\mathrm{Al}$ elemental powders and milling times $t$ in the range from 12 to $36 \mathrm{~h}$. To

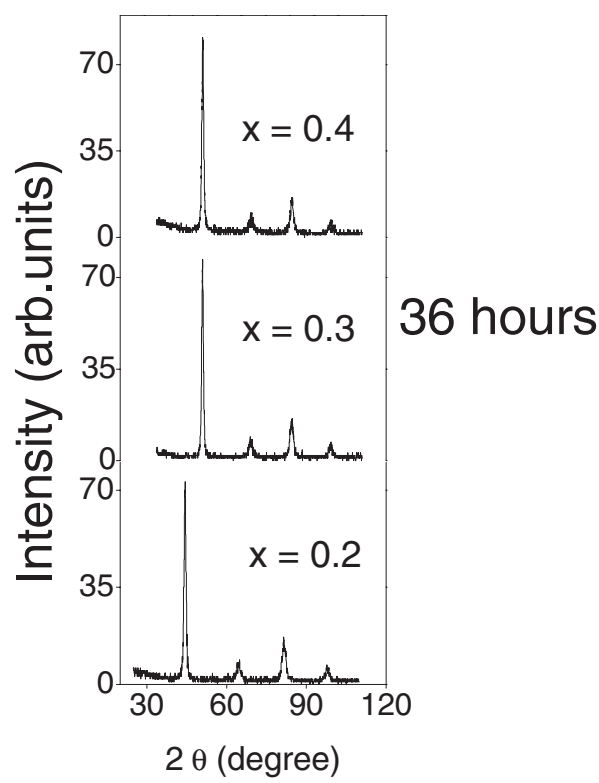

FIG. 1. XRD patterns of samples with $x=0.4,0.3$, and 0.2 milled for $36 \mathrm{~h}$.

avoid overheating and oxidation, the process was performed evacuating the jars and then alternating $1 \mathrm{~h}$ milling followed by $1 \mathrm{~h}$ resting. The powders were mixed at the stoichiometric compositions and then sealed in previously evacuated stainless steel jars of a Pulverisette 5 ball mill system equipped with balls of the same material. A ball mass to powder mass relation of 15:1 was used. After the milling process, the samples were characterized by XRD using a Siemens D 5000 diffractometer and $\mathrm{Cu} K \alpha$ radiation with angular steps of $0.02^{\circ}$ and a step time of $2 \mathrm{~s}$. The patterns were refined by the Rietveld method using the GSAS program. ${ }^{29}$ Energy dispersive x-ray (EDX) compositional maps were recorded by using a JEOL scanning electron microscopy (SEM). Mössbauer spectra were collected in the transmission mode with a Co-57(Rh) source and fitted with the MOSFIT program ${ }^{30}$ taking an $\alpha$-Fe foil as the calibration sample. Hysteresis cycles were recorded using a VSM of Oxford Instruments with a maximum field of $1.5 \mathrm{~T}$. The ac magnetic susceptibility scans were obtained by means of a MAGLAB system of Oxford Instruments with an ac field of $30 \mathrm{Oe}$ at frequencies of $100,316,562$, and $1000 \mathrm{~Hz}$.

\section{EXPERIMENTAL RESULTS AND DISCUSSION}

Figure 1 shows the XRD patterns recorded from the samples milled for $36 \mathrm{~h}$. The patterns of samples milled for other times are very similar and all of them present exclusively the characteristic peaks of a bcc structure. From the Rietveld refinement of the diffraction data both the lattice parameter and the average grain size were calculated and Figs. 2(a) and 2(b) show the variation in those parameters with the milling time. The possible presence of microstresses on the particles was taken into account on the Rietveld refinement of the XRD patterns. In Fig. 2(a) it can be observed that the lattice parameter obtained in the samples with $x$ $=0.3$ and 0.4 is nearly independent of the milling time, while 

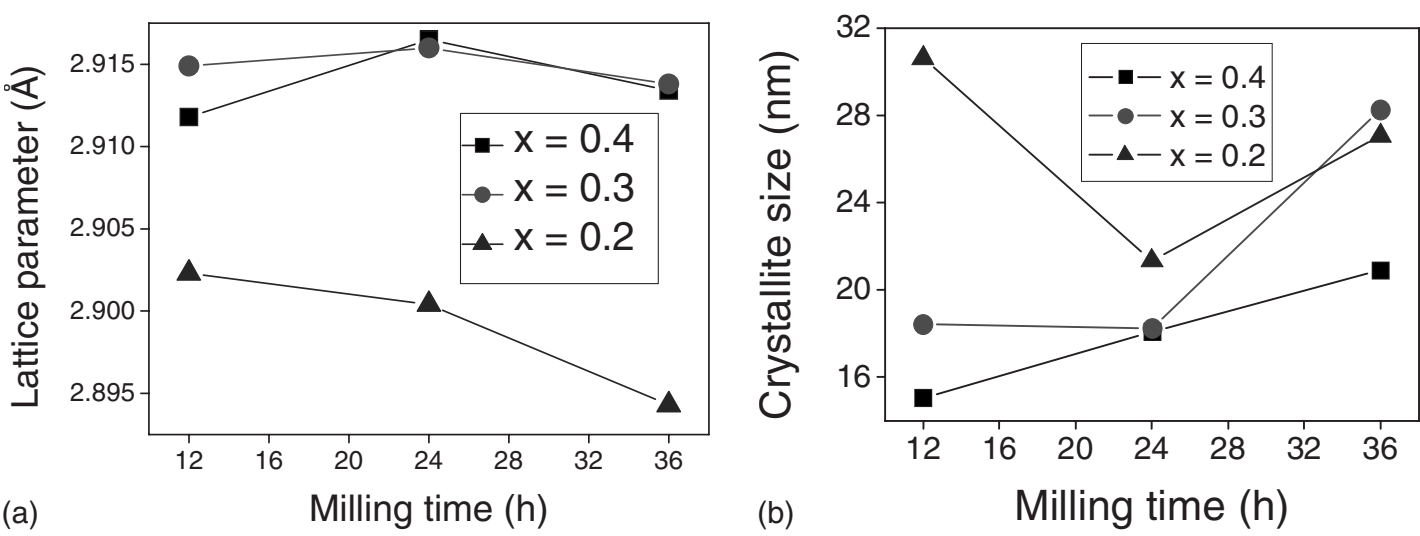

FIG. 2. (a) Lattice parameter and (b) mean crystallite size as a function of the milling time for the different prepared samples. Error bars are smaller than depicted symbols in all cases.

that measured in the $x=0.2$ sample decreases from $2.903 \AA$ (12 h milling) down to $2.894 \AA$ (36 h milling).

The behavior shown in Fig. 2(a) can be understood if we take into account that the $\mathrm{Al}$ atoms enter the Fe lattice producing a local dilatation due to their larger size. According to the literature ${ }^{2,4}$ the alloys having reduced $\mathrm{Al}$ content do not experience any lattice expansion until the $\mathrm{Al}$ at. \% reaches values of the order of $x=0.1$. At these compositions the local dilatations become correlated and the lattice presents a global expansion with $\mathrm{Al}$ content until a concentration of about $x=0.25$; over this concentration the dependence of lattice parameter with $\mathrm{Al}$ content is clearly reduced and remains nearly constant, though contradictory results can be found on the literature, ${ }^{17,24,28}$ reporting very small expansions and contractions. These results are in good agreement with the behavior of samples with $x=0.3$ and 0.4 , which present similar $a$ values. Particularly, the $a$ value measured in the $12 \mathrm{~h}$ milled sample is indistinguishable from those observed in the samples having the same composition and milled for longer times which reveals that after just $12 \mathrm{~h}$ milling the bcc lattices are already formed (except for sample with $x=0.4$ and $t=12 \mathrm{~h}$ for which a small quantity of Fe was detected by SEM/EDX, lest than $3 \%$ in volume, see below). For the samples with $x=0.2$ the bcc lattice is already present after $12 \mathrm{~h}$ milling as evidenced by the measured lattice parameter which is larger than that of pure Fe (but smaller than those of $x=0.3$ and 0.4 samples) and shows that the local expansions are already correlated.

As it can be noted in Fig. 2(b) the mean crystallite size shows a trend to increase as the milling time increases, except for samples with $x=0.2$. Those milling time dependencies of the average grain size reflect the complex interplay between the fracturing and soldering processes taking place jointly with the large plastic deformations experienced by the alloy powders during the milling progression. Also, they reflect the fact that the ductility of the Fe rich powders is here balanced by the larger fragility of those containing the larger amounts of $\mathrm{Al}$.

Figure 3 shows the room temperature Mössbauer spectra recorded from the three different alloys milled for $36 \mathrm{~h}$. These spectra show broad and asymmetric lines characteristic of disordered ferromagnetic systems ${ }^{31}$ and, consequently, they were fitted using hyperfine magnetic field distributions
(HMFDs). The spectra recorded from the samples milled for shorter times were very similar to those depicted in Fig. 3, except for those recorded from the alloy with $x=0.4$ milled for 12 and $24 \mathrm{~h}$ which present an additional paramagnetic singlet (see below).

Inspection of Fig. 3 indicates that the spectra recorded from the different alloys and the HMFDs used to fit them show clear differences with composition. In general, it can be stated that the lines of the spectra (and therefore their corresponding HMFDs) are broader as Al concentration increases. While the distribution corresponding to the sample with $x=0.2$ is nearly symmetric, the distributions corresponding to the other two samples show a noticeable contribution in the region of small hyperfine magnetic field values. This is especially evident in the sample with $x=0.4$. Consequently, the average hyperfine magnetic field values deduced from the different distributions decrease with increasing $\mathrm{Al}$ content $(28,24$, and $23 \mathrm{~T}$ for $x=0.2,0.3$, and 0.4 , respectively). If these $\left\langle B_{\mathrm{hf}}\right\rangle$ values are compared with those corre-
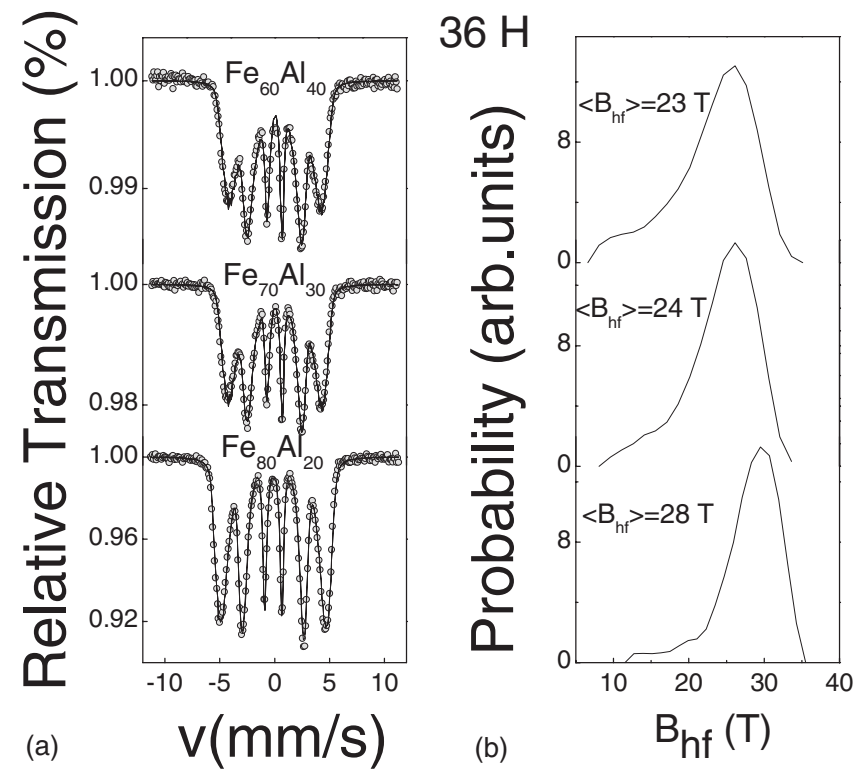

FIG. 3. (a) Mössbauer spectra and (b) their corresponding HMFDs, taken at RT on samples with $x=0.4,0.3$, and 0.2 , milled for $36 \mathrm{~h}$. 


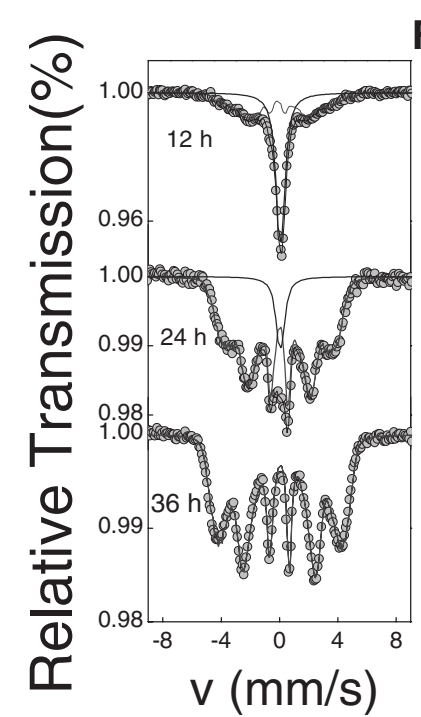

$\mathrm{Fe}_{60} \mathrm{Al}_{40}$

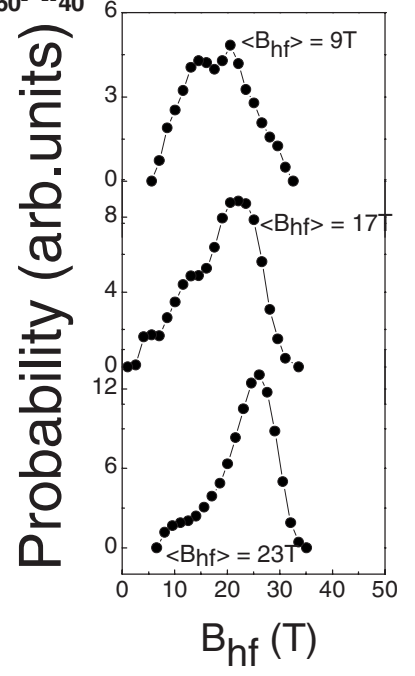

FIG. 4. Mössbauer spectra at RT and their corresponding HMFDs of samples with $x=0.4$ and milled for 12, 24, and $36 \mathrm{~h}$, respectively.

sponding to disordered alloys of the same composition but prepared by melting [namely, 28, 22, and $19 \mathrm{~T}$ (Ref. 24)] one finds that, except for the alloy with $x=0.2$, the field values are larger when the materials are produced by MA than when they are produced by melting. Particularly different is the value found for the $x=0.4$ alloy, that is, the one having the largest $\mathrm{Al}$ content. It is also interesting to note that the HMFDs of melted and disordered samples of the same composition than those studied here present additional peaks whose intensities and number increase when the $\mathrm{Al}$ concentration grows. To interpret these differences it is necessary to remember that the presence of peaks in the HMFD is an indication that some local configurations around the Fe sites are more probable than others or, in other words, that some specific or preferential local orders are present. Then, when the HMFD is smooth and shows just one maximum it means that the probability of finding some of these more probable local configurations is smaller. In the present case this would mean that the distribution of $\mathrm{Al}$ atoms around $\mathrm{Fe}$ is at random around just one more probable local configuration. Thus, the disorder is larger and this disorder increases $\left\langle B_{\mathrm{hf}}\right\rangle$ and consequently the ferromagnetism.

In order to follow in further detail the compositional evolution of the samples during the milling process, Mössbauer spectra at RT were recorded from samples with $x=0.4$ milled for 12,24 , and $36 \mathrm{~h}$ (Fig. 4). The MS corresponding to the sample milled for $t=12 \mathrm{~h}$ was fitted with a HMFD, a singlet and a small sextet. This sextet corresponds to $\alpha$-Fe particles which are not alloyed or that contain a very reduced $\mathrm{Al}$ amount (the presence of those particles was confirmed by SEM/EDX compositional maps, see Fig. 5). The small concentration of $\alpha$ - Fe (the relative area of the $\alpha$-Fe sextet in the Mössbauer spectra was approximately $1.5 \%$ ) prevented its identification by XRD. The MS of the sample with $t=24 \mathrm{~h}$ was fitted considering a HMFD and a singlet, whereas that corresponding to the $t=36 \mathrm{~h}$ sample was fitted using a HMFD exclusively. As it can be observed in Fig. 4 the $\alpha$-Fe

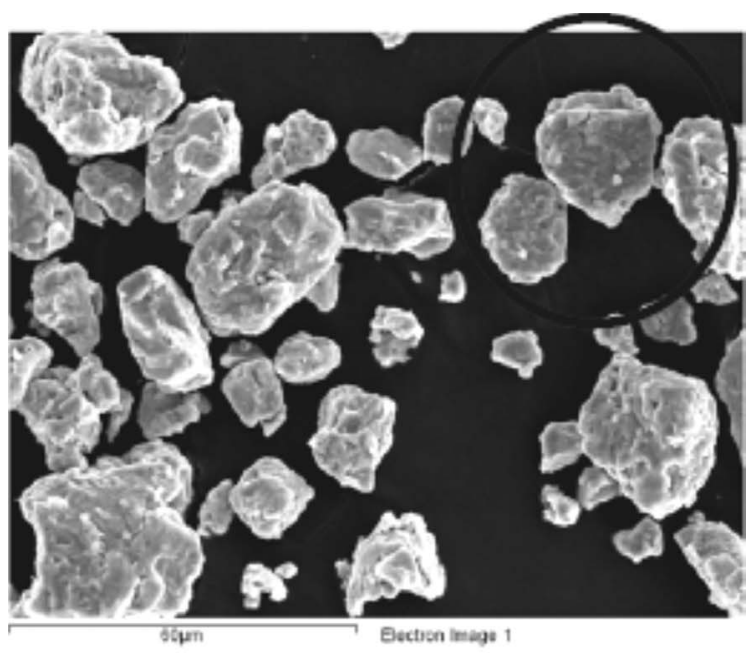

(a)

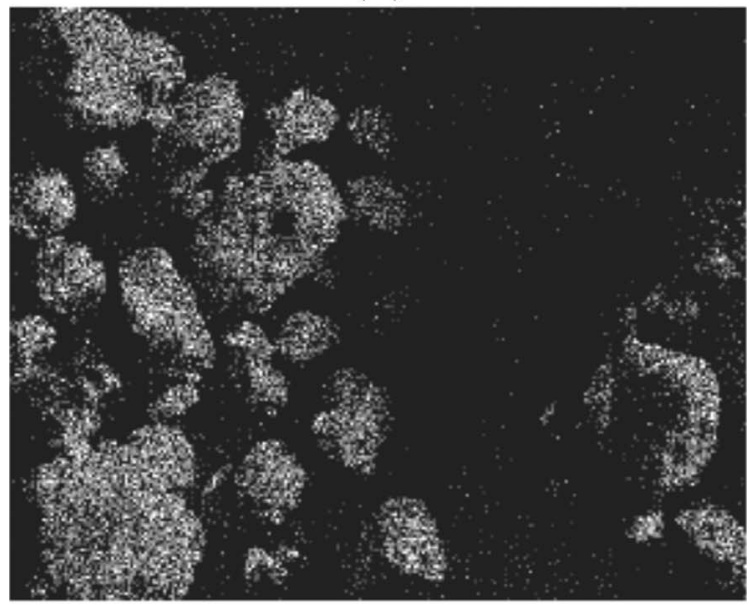

, $\mathrm{Ka1}$

(b)

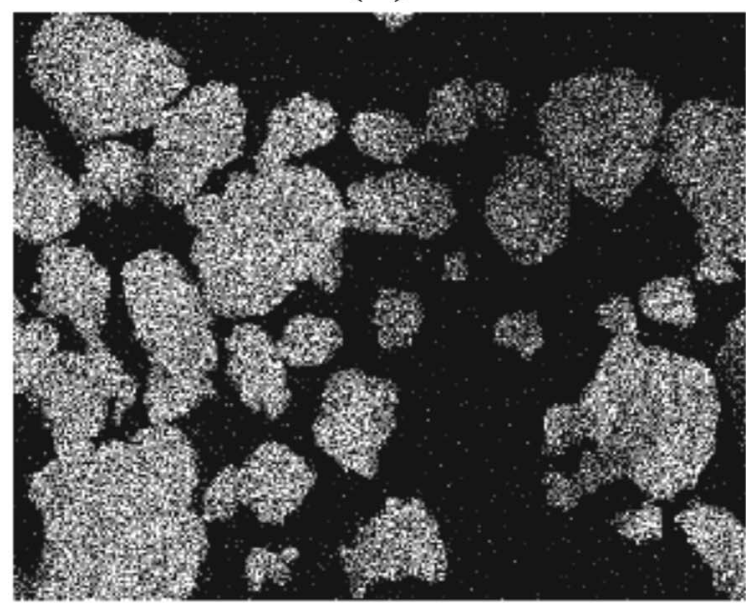

(c)

FIG. 5. (a) SEM picture and [(b) and (c)] EDX compositional maps of a region of the $\mathrm{Fe}_{60} \mathrm{Al}_{40}$ sample milled for $12 \mathrm{~h}$ in which some particles of almost pure $\alpha$-Fe are detected. Note that (a) the particles enclosed within the red circle appear in black in the $\mathrm{Al} \mathrm{K \alpha}$ map and (c) they are bright in the Fe $K \alpha$ map, indicating that they are rich in $\mathrm{Fe}$ and poor in $\mathrm{Al}$. 


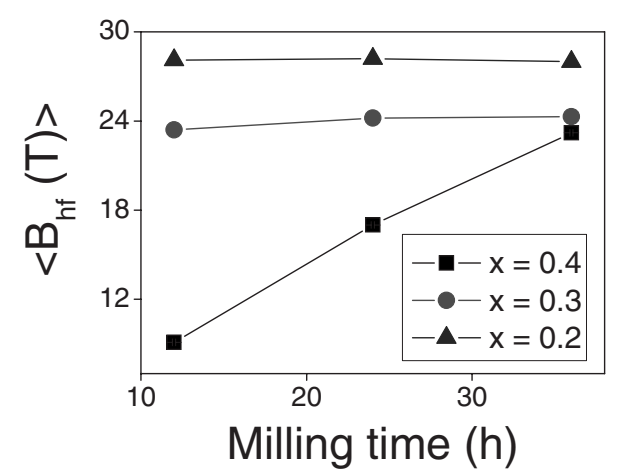

FIG. 6. Variation in the mean hyperfine field with the milling time for samples with $x=0.4,0.3$, and 0.2. Error bars are smaller than depicted symbols.

sextet is not present in the samples milled for $t=24$ and $36 \mathrm{~h}$ indicating that for these milling times the original Fe powders are completely alloyed. Figure 4 also shows that the relative intensity of the paramagnetic line decreases with the increase in the milling time, being already absent in the spectrum of the sample milled for $t=36 \mathrm{~h}$. The HMFD of the sample milled for $t=12 \mathrm{~h}$ presents a $\left\langle B_{\mathrm{hf}}\right\rangle$ of $9 \mathrm{~T}$, and two principal peaks at 14 and $20 \mathrm{~T}$; that milled for $t=24 \mathrm{~h}$ presents a $\left\langle B_{\mathrm{hf}}\right\rangle$ of $17 \mathrm{~T}$, a principal peak at $22 \mathrm{~T}$, and a secondary one at $12 \mathrm{~T}$. Finally, for $t=36 \mathrm{~h}\left\langle B_{\mathrm{hf}}\right\rangle$ is $23 \mathrm{~T}$ and the HMFD presents basically a single peak at $26 \mathrm{~T}$, having an almost symmetric shape. The results suggest that the milling time stabilizes the ferromagnetic order of the sample and this fact is associated to the increase in the disorder in the system. The increase in disorder with increasing milling time in the mechanically alloyed Fe-Al system has been observed by different authors ${ }^{11,17-22,32}$ and in our case is evidenced by the fact that the peaks corresponding to the different more probable local configurations around the $\mathrm{Fe}$ sites progressively disappear with the increasing milling time and the HMFD shows just one maximum and a symmetric shape around the most probable Fe site for $t=36 \mathrm{~h}$.

Figure 6 shows the evolution of $\left\langle B_{\mathrm{hf}}\right\rangle$ as a function of the milling time for the studied samples. It can be noted that, as it was expected, samples with larger $\mathrm{Fe}$ content present a higher $\left\langle B_{\mathrm{hf}}\right\rangle$ and that this field is larger for longer milling times. This behavior is more notable for the sample richest in Al. As it was discussed previously, increasing the milling time increases the disorder and consequently the $\mathrm{F}$ behavior. If this behavior is compared with that previously shown by the lattice parameters, it follows that the enhancement of the $\mathrm{F}$ behavior cannot be associated, in the current case, with the increase in the lattice parameter. As it can be seen in Fig. 1 the samples with $x=0.3$ and 0.4 present similar $a$ values for all the milling times; however, the sample with $x=0.3$ presents only a slight increase in $\left\langle B_{\mathrm{hf}}\right\rangle$ while the sample with $x=0.4$ presents a huge increase in $\left\langle B_{\mathrm{hf}}\right\rangle$ value. Besides, the $a$ value for the sample with $x=0.2$ decreases with the milling time, whereas its $\left\langle B_{\mathrm{hf}}\right\rangle$ remains nearly constant, showing that in the present case the enhancement of the $\mathrm{F}$ behavior is not associated to lattice expansion but, more likely, related to disorder and dilution.

Figure 7 shows the hysteresis cycles obtained at RT from the different samples with $x=0.2$ and 0.4. As it can be ob-
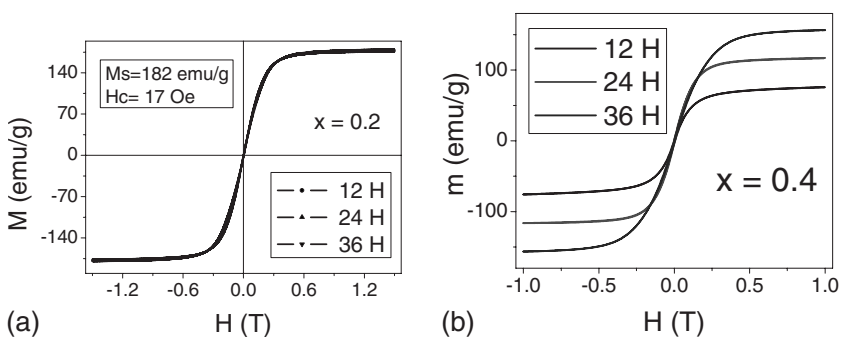

FIG. 7. Hysteresis cycles of samples (a) with $x=0.2$ and (b) with $x=0.4$.

served in that figure, the cycles saturate near $0.5 \mathrm{~T}$. For the sample with $x=0.2$ the cycles [Fig. 7(a)], despite the milling time dependencies of the lattice parameter and average grain size, are practically milling time independent, with a saturation magnetization $\left(M_{s}\right)$ of approximately $182 \mathrm{emu} / \mathrm{g}$ and a coercive field $\left(H_{c}\right)$ of 17 Oe. However, large differences can be observed among the cycles measured in the samples with $x=0.4$ [Fig. 7(b)]. In this case the high-field magnetization value increases markedly with the milling time which as already mentioned is in correspondence with the RT stabilization of the ferromagnetic order. From these cycles, and those recorded from the $x=0.3$ sample (not shown), it was possible to determine $M_{s}$ and $H_{c}$ as a function of the milling time. The corresponding results are plotted in Fig. 8 and are in clear correspondence with the milling time evolution of $\left\langle B_{\mathrm{hf}}\right\rangle$ shown in Fig. 6.

Considering the interesting behavior exhibited by the samples with $x=0.4$, we recorded MS at different temperatures between 15 and $298 \mathrm{~K}$ from the sample milled for $t$ $=12 \mathrm{~h}$ (Fig. 9). As it was mentioned previously, the RT spectrum of this sample was fitted with a HMFD, a small sextet characteristic of $\alpha$-Fe and a broad singlet. This fitting model was adopted for the rest of spectra recorded at lower temperatures. Figure 10 presents the variation with temperature of $\left\langle B_{\mathrm{hf}}\right\rangle$ of the resulting HMFD and evidences the occurrence of two anomalies in that temperature dependence taking place near 160 and $65 \mathrm{~K}$, respectively. The spectral area associated with the $\alpha$-Fe sextet remains nearly constant with $T$ and presents values between $1.5 \%$ and $2 \%$.

In order to explore the origin of this phenomenology complementary measurements were conducted. In Fig. 11 we have plotted the temperature variation in the imaginary part of the ac susceptibility of $x=0.4,12 \mathrm{~h}$ milled sample, ob-
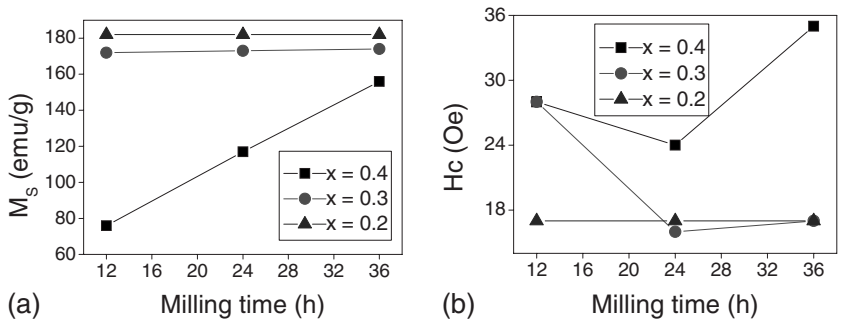

FIG. 8. Variation in the (a) $M_{s}$ and (b) $H_{c}$ values with the milling time for the prepared samples. Error bars are smaller than depicted symbols. 

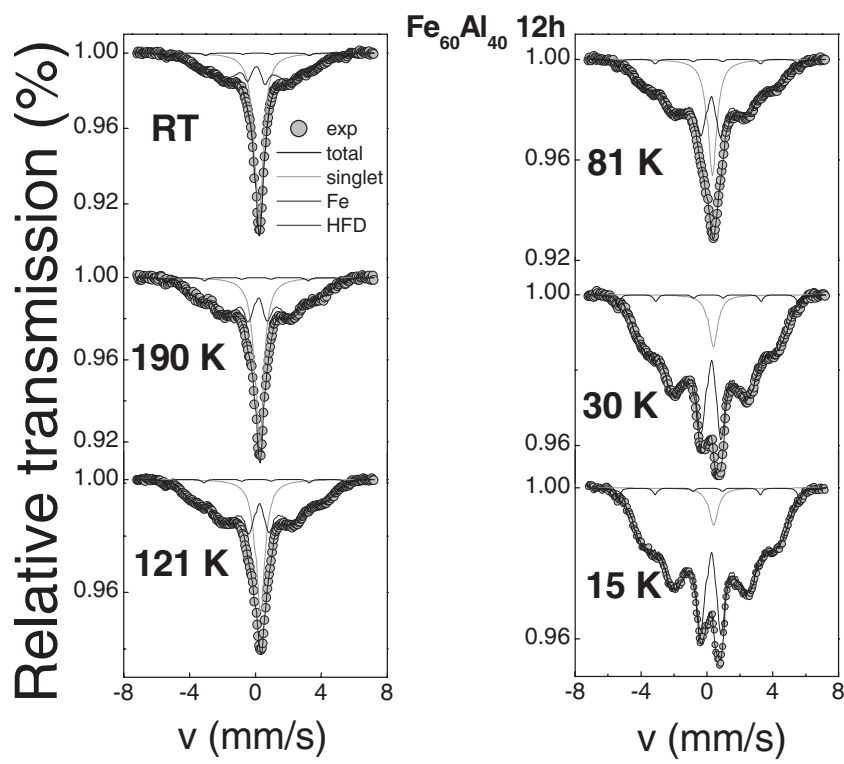

FIG. 9. Mössbauer spectra taken at different temperatures on the sample with $x=0.4$ and milled for $12 \mathrm{~h}$.

tained for 30 Oe peak value ac fields having frequencies $f$ in the range from 100 up to $1000 \mathrm{~Hz}$. It is clear from that figure that two maxima appear, the first one occurring at a temperature which varies, with the variation in $f$, from 85 to $89 \mathrm{~K}$. The second maximum takes place at temperatures varying with the frequency from 166 up to $188 \mathrm{~K}$. The weak frequency dependence of the temperature at which the first maximum appears suggests that it is associated to a spinglass freezing transition, ${ }^{33}$ and specifically, and due to the observation of $\mathrm{F}$ order at this temperature, to a re-entrant spin-glass transition. The second maximum in the ac susceptibility dependence on temperature exhibits a much larger frequency dependence ( $22 \mathrm{~K}$ for a frequency decade), which can be related to a blocking temperature of the SP regions present in the sample. ${ }^{34}$ The differences observed between these maxima in the temperature dependence of the ac susceptibility and the anomalies observed in that of $\left\langle B_{\mathrm{hf}}\right\rangle$ can be ascribed mainly to the uncertainty in defining the transition temperature from the curves obtained by Mössbauer spec-

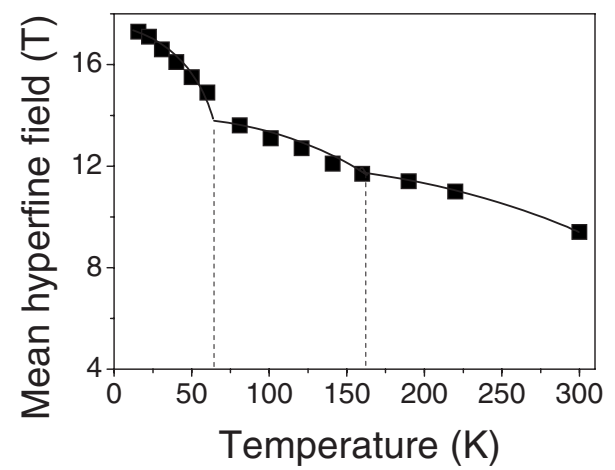

FIG. 10. Variation in the $\left\langle B_{\mathrm{hf}}\right\rangle$ with the temperature for sample with $x=0.4$ and $12 \mathrm{~h}$ of milling. Continuous lines are a guide for eyes; error bars are smaller than depicted symbols.

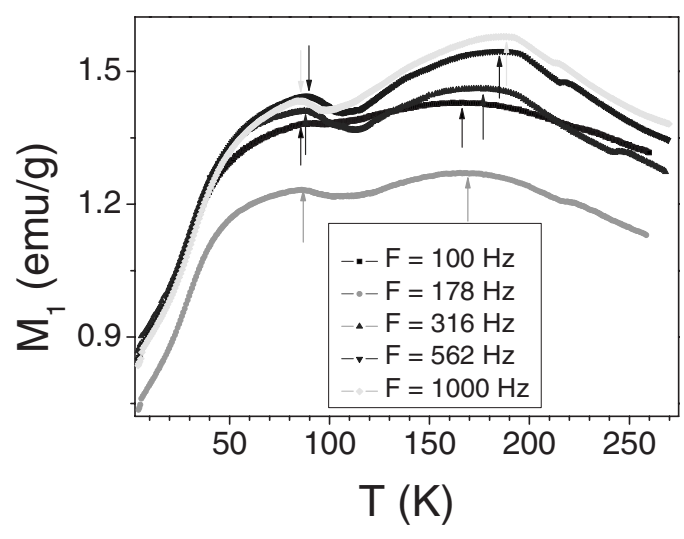

FIG. 11. ac susceptibility (imaginary part) variation with temperature and measuring frequency obtained in the sample with $x$ $=0.4$, milled for $12 \mathrm{~h}$.

trometry which contain a smaller number of points than those measured in the susceptibility curves and of course to the different measurement times of the techniques.

These RSG and SP phenomena can be interpreted as a consequence of the disordered character of the sample and the competitive interactions which are present in the system. The existence of disorder is evidenced by the Mössbauer data and also supported by previously reported results on this type of alloys which indicate that (i) MA produces disordered alloys and (ii) the disorder increases with $t .^{11,17-22,32}$ We assume that the competitive interactions, which were reported in previous papers for ordered and disordered melted samples and MA samples, are due to the F interaction between nearest-neighbor (nn) $\mathrm{Fe}$ atoms and the AF nextnearest-neighbor interaction (nnn) between $\mathrm{Fe}$ atoms. The disordered character of the sample allows us to consider the presence of different types of Fe sites: (i) sites very rich in nn Fe atoms (8 or 7); (ii) sites with a majority or intermediate number of nn Fe atoms $(6,5$, or 4$)$ which, in accordance with the composition of the sample (40 at. \% Al), must be the most probable ones and can be considered to form the percolating matrix associated to the global RT, F behavior of the sample; and (iii) sites with a reduced number of nn Fe atoms (3 or less) which would be responsible for both the lower hyperfine magnetic fields observed in the HMFD and the broad P singlet. Then, it would be plausible to think of the existence of $\mathrm{Fe}$ clusters constituted by spatially close, and magnetically coupled, type (i) or type (ii) Fe sites. The formation of these clusters would imply that they were surrounded by type (iii) sites. This picture would allow explaining previous reports ${ }^{11,12}$ and, more specifically, (i) the large shift in temperature of the second maximum of the ac susceptibility curves appearing between 166 and $188 \mathrm{~K}$ and the kink of the temperature dependence of $\left\langle B_{\mathrm{hf}}\right\rangle$ observed at approximately $160 \mathrm{~K}$ that would be due to the blocking of the larger SP clusters (the further increase in the $\left\langle B_{\mathrm{hf}}\right\rangle$ values with decreasing temperature would be due to the blocking process of those clusters which present smaller sizes and of course to the decrease in the thermal energy), and (ii) the small variation with frequency of the low-temperature peak observed in the ac susceptibility curves and the kink ob- 

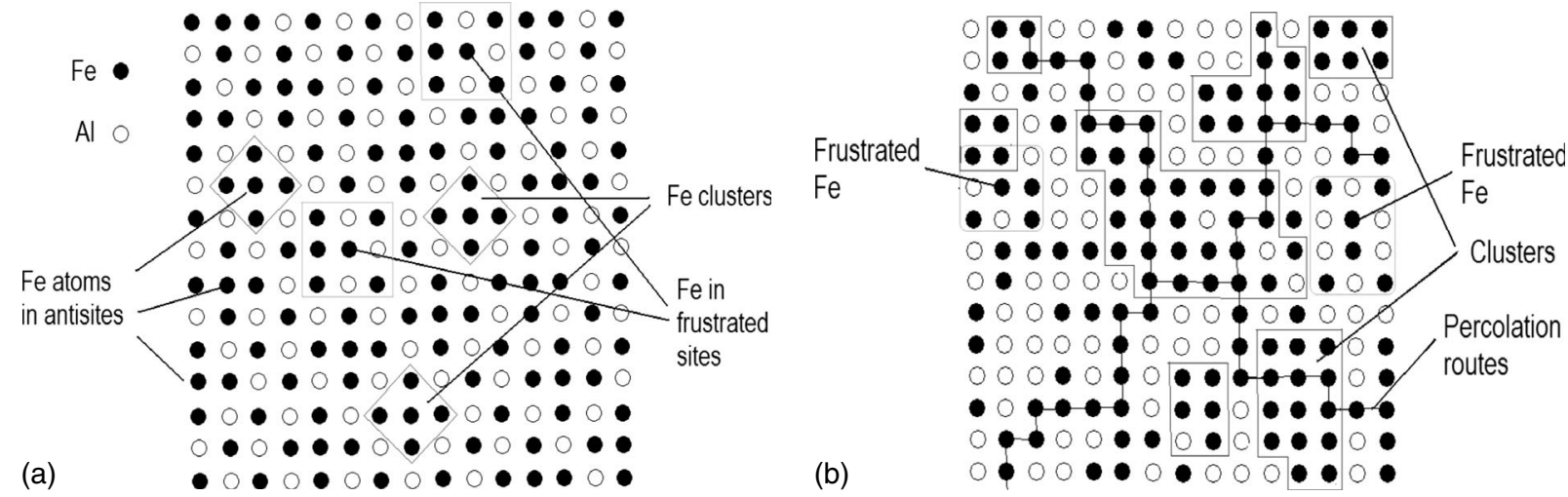

FIG. 12. Square lattice model of $15 \times 15$ atoms for the $\mathrm{Fe}_{60} \mathrm{Al}_{40}$ alloy showing: (a) an ordered sample and (b) a disordered sample.

served at approximately $65 \mathrm{~K}$ in the $\left\langle B_{\mathrm{hf}}\right\rangle$ vs $T$ curve that can be attributed to a RSG behavior. The frustrated spins responsible of the later behavior can be associated to those Fe sites with three or two nn Fe atoms. However, since these sites have a local environment rich in $\mathrm{Al}$ as $\mathrm{nn}$, $\mathrm{AF}$ interactions between nnn $\mathrm{Fe}$ atoms can be also present. In this case, there would exist competition between the F bounds, which are stronger and the AF ones which are weaker but present in a larger amount.

In order to visualize how in ordered and disordered $\mathrm{Fe}_{0.6} \mathrm{Al}_{0.4}$ alloys the frustration and clustering appear, we have plotted in Figs. 12(a) and 12(b) a view of a square lattice of $15 \times 15$ atoms, with $60 \%$ and $40 \% \mathrm{Fe}$ and $\mathrm{Al}$ atoms, respectively, for the case of ordered (a) and disordered (b) alloys (this lattice does not correspond to a real crystalline plane since nn and nnn distances are given by $a$ and $\sqrt{2} a$ but as a picture can be easily extrapolated to the tridimensional case in which the nn and nnn distances are $\sqrt{3} a / 2$ and $a$, respectively). In these figures the black and white circles correspond to $\mathrm{Fe}$ and $\mathrm{Al}$ atoms, respectively. It can be noted in Fig. 12(a) (ordered case) that the Fe atoms in excess $(10 \%)$, compared with the equiatomic composition (50-50), are located in antisites, substituting $\mathrm{Al}$ atoms. This gives place to small Fe clusters (surrounded by small squares) which explains the occurrence of SP clusters ${ }^{10}$ in this type of alloys. The occurrence (surrounded by big squares) of some $\mathrm{Fe}$ sites with just one $\mathrm{Fe}$ as nn (the pair should be coupled through a $\mathrm{F}$ exchange) and having four $\mathrm{Fe}$ nnn pairs (which should be antiferromagnetically coupled) have been also depicted in Fig. 12(a). These competitive magnetic exchange interactions give place to a frustrated site. The occurrence of magnetic frustration, the existence of disorder linked to the nonstoichiometry, and the presence of defects in the lattice would be the origin of the spin-glass phenomena observed at low temperatures ${ }^{4,5}$ in ordered Fe-Al alloys with compositions around 30-40 at. \% Al. Finally, Fig. 12(a) shows how the local magnetic order does not percolate, which implies a global paramagnetic behavior. In Fig. 12(b) (disordered case) it can be noted that the structural disorder allows, in contrast with the ordered case, the formation of clusters of different sizes and shapes (polygons with a majority of black points inside). That distribution can be linked to the observation of a broad peak in the ac susceptibility curves originated by the distribution of SP blocking temperatures associated to the cluster size distribution. In the disordered case the frustrated sites also appear (as indicated in the figure), and they can have different shapes and sizes. The most important fact observed in the disordered picture is the occurrence of percolation paths of ferromagnetically coupled local sites, explaining the close relationship between compositional disorder and magnetic order. Finally our picture also accounts in the disordered case for the observation of RSG transitions since in this case both percolating $\mathrm{F}$ ordered sites and frustrated ones coexist.

\section{CONCLUSIONS}

In this work we have reported on the magnetic and structural properties of the $\mathrm{Fe}_{1-x} \mathrm{Al}_{x}$ alloys $(0.2 \leqslant x \leqslant 0.4)$ system produced by mechanical alloying and milled for $t=12,24$, and $36 \mathrm{~h}$. All the samples present a bcc lattice with a disordered structure and are F at RT independently of the milling time. The structural and magnetic properties of the alloys with $x=0.2$ and 0.3 do not show important variations with $t$, while those of the more diluted one $(x=0.4)$ are strongly dependent on the milling time. For this latter alloy it was found that the lattice parameter does not change significantly with the milling time, while the ferromagnetic behavior is enhanced and becomes comparable to that of the alloys with $x=0.2$ and 0.3 when $\mathrm{t}$ increases. Magnetic measurements prove the existence of re-entrant spin-glass and superparamagnetic phenomena in the sample with $x=0.4$ milled for $t$ $=12 \mathrm{~h}$ with an $\alpha$-Fe content of 1.5 at. $\%$ in the alloy. Thus, the stabilization in these samples of the F order up to temperatures above RT and the observation of RSG transitions and of SP blocking can be interpreted in terms of a simple localized disordered model which accounts for the observation of global F order clustering and the occurrence of frustrated local neighborhoods, namely, those where ferromagnetic nn Fe-Fe interactions and antiferromagnetic nnn Fe-Fe interactions are present. Maybe our more important conclusion is that for the particular $x=0.4 \mathrm{MA}$ alloyed samples the stabilization of the ferromagnetic order takes place exclusively through the induction of compositional disorder and without any contribution from the lattice expansion. 


\section{ACKNOWLEDGMENTS}

The authors would like to thank the financial support given to this work by Colciencias-CNPq and ColcienciasCSIC (Colombian, Brazilian, and Spanish agencies) across collaboration projects. G.A.P.A., the Sabbatical Year Grant provided by the Spanish Science Ministery and the Universidad del Valle. Financial support from the Comunidad Autónoma de Madrid through Project No. S-0505/MAT/0194 is also gratefully acknowledged. Special thanks are given to J. A. Plascak from UFMG, Brazil, for helpfully reading the paper and for his suggestions.
${ }^{1}$ G. P. Huffman and R. M. Fisher, J. Appl. Phys. 38, 735 (1967).

${ }^{2}$ I. Vincze, Phys. Status Solidi A 7, K43 (1971).

${ }^{3}$ A. Arrot and H. Sato, Phys. Rev. 114, 1420 (1959).

${ }^{4}$ M. Shiga and Y. Nakamura, J. Phys. Soc. Jpn. 40, 1295 (1976).

${ }^{5}$ G. S. Grest, Phys. Rev. B 21, 165 (1980).

${ }^{6} \mathrm{Ji}$ Chi, Yang Li, F. G. Vagizov, V. Goruganti, and Joseph H. Ross, Jr., Phys. Rev. B 71, 024431 (2005).

${ }^{7}$ R. D. Shull, H. Okomoto, and P. A. Beck, Solid State Commun. 20, 863 (1976).

${ }^{8}$ W. Bao, S. Raymond, S. M. Shapiro, K. Motoya, B. Fåk, and R. W. Erwin, Phys. Rev. Lett. 82, 4711 (1999).

${ }^{9}$ C. S. Lue, Y. Öner, D. G. Naugle, and J. H. Ross, Jr., Phys. Rev. B 63, 184405 (2001).

${ }^{10}$ J. W. Cable, L. David, and R. Parra, Phys. Rev. B 16, 1132 (1977).

${ }^{11}$ D. S. Schmool, E. Araújo, M. M. Amado, M. Alegría Feio, D. Martin Rodríguez, J. S. Garitaonandia, and F. Plazaola, J. Magn. Magn. Mater. 272, 1342 (2004).

${ }^{12}$ D. Martín Rodríguez, F. Plazaola, and J. S. Garitaonandia, Hyperfine Interact. 169, 1231 (2006).

${ }^{13}$ J. Bogner, W. Steiner, M. Reissner, P. Mohn, P. Blaha, K. Schwarz, R. Krachler, H. Ipser, and B. Sepiol, Phys. Rev. B 58, 14922 (1998).

${ }^{14}$ B. V. Reddy, S. N. Khanna, and S. C. Deevi, Chem. Phys. Lett. 333, 465 (2001).

${ }^{15}$ E. Apiñaniz, F. Plazaola, and J. S. Garitaonandia, J. Magn. Magn. Mater. 272-276, 794 (2004).

${ }^{16}$ G. P. Das, B. K. Rao, P. Jena, and S. C. Deevi, Phys. Rev. B 66, 184203 (2002).

${ }^{17}$ D. A. Eelman, J. R. Dahn, G. R. Mackay, and R. A. Dunlap, J. Alloys Compd. 266, 234 (1998).

${ }^{18}$ S. Gialanella, X. Amils, M. D. Barò, P. Delcroix, G. Le Caer, L.
Lutterotti, and S. Suriñach, Acta Mater. 46, 3305 (1998).

${ }^{19}$ A. Hernando, X. Amils, J. Nogués, S. Suriñach, M. D. Barò, and M. R. Ibarra, Phys. Rev. B 58, R11864 (1998).

${ }^{20}$ E. Apiñaniz, F. Plazaola, J. S. Garitaonandia, D. Martin, and J. A. Jimenez, J. Appl. Phys. 93, 7649 (2003).

${ }^{21}$ D. Martin Rodríguez, E. Apiñaniz, F. Plazaola, J. S. Garataoandia, J. A. Jiménez, D. S. Schmool, and G. J. Cuello, Phys. Rev. B 71, 212408 (2005).

${ }^{22}$ J. Nogués, E. Apiñaniz, J. Sort, M. Amboage, M. d'Astuto, O. Mathon, R. Puzniak, I. Fita, J. S. Garitaonadia, S. Suriñac, J. S. Muñoz, M. D. Barò, F. Plazaola, and F. Baudelet, Phys. Rev. B 74, 024407 (2006).

${ }^{23}$ A. Taylor and R. M. Jones, J. Phys. Chem. Solids 6, 16 (1958).

${ }^{24}$ G. A. Pérez Alcázar and E. Galvão da Silva, J. Phys. F: Met. Phys. 17, 2323 (1987).

${ }^{25}$ J. H. Hsu and C. L. Chien, Hyperfine Interact. 69, 451 (1991).

${ }^{26}$ A. Fnidiki, J. P. Eymery, and J. Delafond, J. Magn. Magn. Mater. 40, 130 (1983).

${ }^{27}$ H. Bakker, G. F. Zhou, and H. Yang, Prog. Mater. Sci. 39, 159 (1995).

${ }^{28}$ A. Taylor and R. M. Jones, J. Phys. Chem. Solids 6, 16 (1958).

${ }^{29}$ A. C. Larson and R. B. Von Dreele, General Structure Analysis System (GSAS), Los Alamos National Laboratory Report No. LAUR 86-748, 2004.

${ }^{30} \mathrm{~F}$. Varret and J. Teillet, MOSFIT program.

${ }^{31}$ B. Window, J. Phys. E 4, 401 (1971).

${ }^{32}$ E. Jartych, J. K. Zurawics, D. Oleszak, and M. Pȩkala, J. Phys.: Condens. Matter 10, 4929 (1998).

${ }^{33}$ J. A. Mydosh, Spin Glasses: An Experimental Introduction (Taylor \& Francis, London, 1993), p. 86.

${ }^{34}$ D. Weller and A. Moser, IEEE Trans. Magn. 35, 4423 (1999). 